\title{
Estrategias y recursos de información para la gerencia de proyectos compartidos y sectoriales: el sector pesquero artesanal de las Azores
}

\author{
Judite Marieta Canha Fernandes \\ Instituto de Acção Social (Portugal)
}

\section{Resumen}

Se presentan la filosofía y la metodología del proyecto Estrategias y recursos de información para la gerencia de proyectos compartidos y sectoriales, llevado a cabo en el marco del programa comunitario Equal entre septiembre de 2002 y diciembre de 2004, así como los productos desarrollados en él. Se trata de un proyecto de gestión de la información para el desarrollo del sector pesquero artesanal de las Azores (Portugal), cuyos objetivos principales fueron contribuir a la valorización y modernización del sector, crear alternativas de renta, fomentar un aprendizaje continuo y luchar contra las discriminaciones y desigualdades.

Palabras clave: Gestión de la información. Proyectos sectoriales. Proyectos compartidos. Desarrollo social y comunitario. Sector pesquero. Azores (Portugal).

\begin{abstract}
The philosophy, methodology and results of the project Information strategies and resources for managing shared and sectorial projects are presented and discussed. This project was developed in the frame of the European Union EQAUL programme from 2002 to 2004. It was oriented towards the management of information for the development of the craftsman-fishing sector of the Azores (Portugal). Its main aims were to contribute to the industry modernization and revalorization, to create new sources of income, to promote continuous education and learning, and to combate discriminations and inequalities.
\end{abstract}

Keywords: Information management. Shared and sectorial projects. Development. Fishing. Azores (Portugal).

\section{Introducción}

La familia de productos Estrategias y recursos de información para la gerencia de proyectos compartidos y sectoriales fue desarrollada durante la acción 2 del proScire. $14: 1$ (en.-jun. 2008) 75-87. ISSN 1135-3716. 
yecto Mudança de Maré. Este proyecto, orientado al desarrollo del sector pesquero artesanal de las Azores (Portugal), tenía como objetivos principales contribuir a la valorización y modernización del sector, crear alternativas de renta, fomentar un aprendizaje continuo y luchar contra discriminaciones y desigualdades. Fue en este contexto y en función de estos objetivos donde esta familia de productos ha sido desarrollada y probada. Decimos familia de productos porque es un sistema de soluciones complementarias (que también funcionan aisladas); información para la gerencia, porque estas estrategias y recursos apuntan hacia la racionalización y la eficacia del fenómeno social de la información en estos contextos; proyectos compartidos, porque fue concebida especialmente para los proyectos en partenariado y/o red; sectorial, porque las estrategias y los recursos que se consideran aquí se diseñan especialmente para un sector económico.

El trabajo en red se afirma, cada vez más, como capaz de traer nuevas respuestas en el dominio de la cohesión social y del desarrollo sostenible. En la práctica se expresa en un aprendizaje donde las personas participantes se constituyen como comunidad, donde cambian experiencias, compaginan ideas y apoyan mutuamente la resolución de problemas. Sin embargo, esta no es una opción fácil. Los partenariados acertados requieren una nueva cultura, nuevas capacidades y nuevas prácticas de cooperación, sin olvidarse del componente clave de la formación continua. Las dificultades aumentan cuando esos partenariados implican a gente y a instituciones con diversas localizaciones geográficas. La discontinuidad territorial acentúa el esfuerzo de las entidades socias en la confrontación de ideas, el intercambio de experiencias y la resolución de los problemas y las preguntas relacionados con el planeamiento y la gerencia del trabajo y los recursos humanos, y obliga fuertemente a una nueva cultura de información para la gerencia eficaz de estos proyectos.

\section{Entorno teórico y social}

En este proyecto se considera gestión de la información (o del conocimiento explícito) un segmento del objeto de la ciencia de la información, asumiendo desde luego dos referencias de base: información como conjunto estructurado de representaciones mentales codificadas (símbolos significantes), socialmente contextualizadas y capaces de ser registradas en cualquier medio (papel, película, banda magnética, disco compacto, etcétera), comunicadas de forma no sincronizada y multidireccionada (Ribeiro y Silva, 2004); ciencia de la información como la materia que investiga las propiedades y el comportamiento de la información, las fuerzas que dirigen el flujo de esta y los medios de procesamiento de la misma para la optimización de su acceso y uso (Borko, 1968), asumida como un campo del saber y transdisciplinar, inscrito en el área de las ciencias sociales y humanas. Así, el modelo para el proceso de gestión de información debe abarcar toda la cadena de valor de la información, empezando por la identificación de las necesidades de infor-

Scire. $14: 1$ (en.-jun. 2008) 75-87. ISSN 1135-3716. 
mación, pasando por la adquisición, organización y preservación, productos y servicios, y distribución de la información, y cerrando el ciclo con la utilización de la información (Choo, 2003).

En el ámbito de las diversas iniciativas comunitarias, donde los objetivos tienen como eje central procurar encontrar soluciones comunes para problemáticas que se manifiestan en todo el territorio europeo, y que en el Tercer Cuadro Comunitario de Apoyo se materializan en las iniciativas Interreg III, Equal, Leader+ y Urban II, se desarrollan proyectos que engloban entidades, casi siempre organizadas en partenariados o redes locales, nacionales, transnacionales o transfronterizas, y personas, agentes de los proyectos, además de los conocidos objetivos, a quién se dirigen y su razón de existencia. Implican no solo una gran complejidad en sus actividades, sino también el requisito de administrar cada vez mayor cantidad y diversidad de información, de forma que responda con eficacia a las necesidades percibidas. Para esos proyectos europeos, y para un cada vez mayor número de organizaciones que desarrollan proyectos en partenariado o en red en otros contextos, se crean realidades de gestión de información específicas, extremamente dinámicas, que implican profundas adaptaciones de las organizaciones y de las personas implicadas en esos proyectos, y sitúan como desafío central construir y profundizar los conceptos y prácticas asociados a una red o un partenariado efectivos. En el caso de la iniciativa comunitaria Equal es especialmente fuerte el enfoque en la práctica del partenariado, así como la construcción de las diversas redes (temáticas, transnacionales, europeas, etcétera) que se presentan como uno de los ejes base de implementación.

Todo esto en un contexto global de sociedad de la información, donde la información y el conocimiento desempeñan papeles centrales y penetran en todas las esferas de la actividad humana. En ese contexto, los sistemas de información desempeñan un papel fundamental en la economía de la información-conocimiento, con el valor añadido de servir como puntos focales que concentran información producida, por naturaleza, de forma dispersa. Se asume en este artículo el concepto de sistema de información integral activa y permanente (SI-AP), que agrega al pragmatismo de la gestión de la información el capital simbólico de la memoria organizacional o institucional, sin el cual no es posible proyectar una matriz científica y retrospectiva para los modelos (Silva, 2004), y que pretende traspasar la visión, insuficiente, del sistema de información como "mera" herramienta tecnológica, sin tener en cuenta la transformación, a diversos niveles, de la propia cultura organizacional, que debe ser subyacente a su construcción y uso eficaz.

Castells (1996), reflexionando sobre el entorno social contemporáneo, lo sintetiza de forma magnífica:

La exageración profética y la manipulación ideológica que caracterizan a la mayor parte de los discursos sobre la revolución de la tecnología de la información no deben llevarnos

Scire. $14: 1$ (en.-jun. 2008) 75-87. ISSN 1135-3716. 
a menospreciar su verdadero significado fundamental. Es [...] un acontecimiento histórico al menos tan importante como lo fue la Revolución Industrial del siglo XVIII, inductor de discontinuidad en la base material de la economía, la sociedad y la cultura. La relación histórica de las revoluciones tecnológicas [...] muestra que todas se caracterizan por su capacidad de penetración en todos los dominios de la actividad humana no como una fuente exógena de impacto, sino como el paño con el que está tejida esa actividad. En otras palabras, se orientan hacia el proceso, además de inducir nuevos productos. Por otra parte, a diferencia de cualquier otra revolución, el núcleo de la transformación que estamos experimentando en la revolución en curso remite a las tecnologías del procesamiento de la información y de la comunicación.

Y esa revolución está pidiendo cambios profundos a las sociedades incluso en una misma generación, en un entorno siempre cambiante. Por eso, las adaptaciones para las personas, en especial para las generaciones mayores, son brutales. Esto puede crear antagonismos en las relaciones con las tecnologías entre generaciones, que corten el proceso de comunicación, creen dobles velocidades y acentúen procesos de infoexclusión (ya antiguos pero que aún no hemos sido capaces de resolver). Esa realidad está presente en estos proyectos (como en la mayoría de los entornos) y tiene que ser trabajada a corto, medio y largo plazo, procurando que el proceso de utilización de las herramientas de gestión de información se desarrolle de forma positiva y democrática y que se evite la "exclusión" de algunos agentes de proyecto del proceso de gestión de información. La discusión y aceptación del modelo de gestión de información con la coordinación del proyecto es un momento crítico, previo a la implementación del sistema de información, que debe ser garantizado y bien trabajado, de forma que asegure su apoyo, identificación con los objetivos y cohesión en el proceso de implementación, en términos de gestión de información.

En los proyectos, como en cualquier organización, es buena y constructiva la articulación entre objetivos, estrategias, metodologías y, en la base de todo ello, personas, que puede marcar la diferencia de calidad. Son las personas las que definen los objetivos y las estrategias, practican las metodologías, se comunican (mejor o peor) entre sí, cumplen las tareas; en fin, ejecutan los proyectos. En el contexto de las prácticas sociales, la información es un elemento fundamental, pues es compartiendo la información como los sujetos sociales comunican y adquieren el conocimiento de sus derechos y deberes, y toman decisiones sobre sus vidas, sea de forma individual o colectiva. Así, participan en circuitos de información/comunicación, en este caso en partenariado o en red; los sujetos sociales construyen prácticas de información cuyo conocimiento también es esencial para el desarrollo de los sistemas de información y de la propia sociedad de la información. Grosso modo: ¿qué prácticas de información y comunicación vamos a desarrollar y necesitamos?, ¿cómo funcionan la recepción, selección, apropiación y transformación de la información disponible a través de los sistemas de información?

Scire. $14: 1$ (en.-jun. 2008) 75-87. ISSN 1135-3716. 
Aquí se considera que estos proyectos en partenariado o red pueden constituir un interesante estudio de caso para un mejor conocimiento de las prácticas contemporáneas de información, en la medida que reúnen todas las variables objeto de estudio (trabajo en proximidad/comunidad pero distanciado físicamente, uso de tecnologías, ambiente dinámico de información y comunicación, necesidad de toma de decisiones rápida y compartida, trabajo en red, etcétera) y, al mismo tiempo, permiten observar, aislar y controlar las variables de forma que se extraigan resultados cualitativos y cuantitativos de calidad.

En síntesis, las cuestiones teóricas y prácticas asociadas a la gestión de información configuran, cada vez más, aspectos cruciales del desarrollo de las sociedades en su conjunto, en las que urge profundizar de forma consistente. En la construcción del "espacio europeo" se asumen particularidades y contextos para las entidades y sujetos que participan en esos proyectos, pero también para las iniciativas en su conjunto, lo que justifica una reflexión profunda que pueda consolidar las prácticas, la democracia y la legibilidad de los procesos, producir los cambios políticos y sociales deseados, aproximar las poblaciones y aportar datos importantes para el desarrollo teórico de la gestión de la información como segmento de la ciencia de la información, en la acepción ya referida. Contribuir a alcanzar todos estos objetivos es lo que se pretende con el proyecto de doctorado Ciencia de la información en la gestión de proyectos de ámbito europeo: la iniciativa comunitaria Equal, empezada en febrero de 2005, donde la autora se propone continuar, profundizar, desarrollar y sistematizar experiencias de trabajo y resultados prácticos obtenidos a lo largo del proyecto Mudança de Maré, descritos en este artículo, utilizando metodologías cualitativas (estudio de caso) y cuantitativas (cuestionario) para extraer resultados teórico-prácticos que puedan ser útiles para el desarrollo de buenas prácticas de gestión de la información en proyectos en red y en partenariado, dentro y fuera del contexto europeo.

\section{Caracterización general del proyecto}

El partenariado del proyecto Mudança de Maré tiene 9 entidades socias nacionales y 4 transnacionales, entidades públicas y privadas organizadas de forma horizontal/democrática, y más de 50 agentes, hombres y mujeres, que participan en el desarrollo de un total de 29 actividades, cada una de las cuales puede ser considerada un proyecto per se, fuertemente articuladas (desde el punto de vista práctico, formal, temático y simbólico), en un contexto de gran dispersión y discontinuidad geográfica como el de las islas Azores, donde se encuentra la mayoría pero no la totalidad de las entidades socias nacionales. Su objetivo, la población pesquera artesanal de las Azores, incluso sus generaciones más jóvenes, está distante de las "nuevas tecnologías". Los agentes del proyecto, más de 50 como hemos comentado anteriormente, constituían un grupo heterogéneo en términos de

Scire. $14: 1$ (en.-jun. 2008) 75-87. ISSN 1135-3716. 
edad, formación, proximidad con las tecnologías (en algunos casos, fobia), contexto y área de trabajo, con una representación equitativa de los dos sexos. Se puede comprender así que, con dos años para ejecutar las actividades (que siempre crecen a medida que se desarrollan), cumplir la metodología, los trámites administrativos y financieros, tomar decisiones y trabajar en conjunto, obtener resultados, etcétera, siempre trabajando con (no para) la población objetivo, resulten críticos los fenómenos sociales de la información y la comunicación, y la eficacia de la gestión de la información.

\section{Metodología y objetivos}

El primer paso para la construcción de esta familia de productos fue la definición del sistema de información del proyecto, hecha a partir de su análisis orgánicofuncional, donde se clarificaron su estructura y sus funciones en el partenariado. Para esta definición hubo que atender a varias cuestiones: $a$ ) comprensión del caso organizacional (análisis de contexto y extracción de objetivos, competencias, tareas); b) construcción del cuadro orgánico-funcional del partenariado o de la red; c) análisis de la información producida (todos los tipos y suportes); $d$ ) análisis de necesidades y definición de los objetivos para la gerencia de la información; e) clarificación de los circuitos y de los flujos de información - construcción del ciclo de información del proyecto; $f$ ) integración del ciclo de información y del cuadro orgánico-funcional; $g$ ) observación de los sistemas y el equipo tecnológico existentes en las entidades socias; $h$ ) definición del sistema de información del proyecto, metodología y fases de implementación; i) configuración, programación e instalación de software, preferentemente software libre; $j$ ) proceso de formación, evaluación y acompañamiento continuado a los/as agentes de proyecto.

Para este artículo, nos detendremos en los puntos $d$ (análisis de necesidades y definición de los objetivos para la gerencia de la información) y $h$ (definición del sistema de información del proyecto).

\subsection{Análisis de necesidades y definición de objetivos}

El sistema de información del proyecto debe alcanzar los objetivos siguientes: a) crear mecanismos estructurantes de información para la gerencia de proyectos en partenariado o red; $b$ ) contribuir al desarrollo de las capacidades y prácticas informativas de los agentes implicados en estos proyectos; $c$ ) contribuir a la valorización de un sector económico a través de la creación de los recursos informativos adaptados a sus necesidades.

Para la construcción de los recursos necesarios para el desarrollo de los objetivos citados arriba se propuso, en este contexto, subdividir (solamente por razones operacionales) el sistema de información del proyecto en sistema de información interno, organizador de la información para la gerencia interna del proyecto, 
y sistema de información externo, organizador de la información cuyos primeros destinatarios son externos al proyecto.

\subsection{Definición del sistema de información del proyecto}

\subsubsection{Sistema de información interno}

El sistema de información interno corresponde, grosso modo, al sistema de información archivo, en este caso pluricelular, descentralizado y concebido en una lógica poscustodial. Dos puntos se consideran críticos: $a$ ) existencia, en el partenariado/red, de un lenguaje común para la gerencia de información (significados, aplicaciones, procedimientos); $b$ ) creación de una herramienta que garantice la gerencia del sistema de información interno como red o partenariado "verdadero".

Para solucionar el primer punto se estableció un plan de clasificación de información, común a todos los socios, que fijaba las rúbricas informativas. Por otra parte, se elaboró un glosario de términos y las orientaciones generales para los procedimientos administrativos, el control del circuito de la correspondencia general, la codificación de la terminología y la afinación de códigos y conceptos para una mayor simplicidad de procesos y una mejor comunicación. Además, se definió la normalización de procedimientos en la producción de la información. Más adelante, con el fin de obtener una dinámica "verdadera" de la gerencia en partenariado o red, basada en el fenómeno de la información, se creó un sistema de administración documental (SAD) en línea en el portal www.pescas.net. Este sistema tiene su base en el plan de clasificación de información y permite crear, organizar, consultar, compartir, recuperar y evaluar la información producida en todo momento o lugar.

\subsubsection{Sistema de información externo}

En términos de información externa se realizó un análisis genérico del panorama informativo del sector estudiado (industrias pesqueras artesanales de las Azores). Estas fueron las conclusiones: $a$ ) no existen recursos informativos dirigidos (o producidos) exclusivamente para el sector; $b$ ) la información disponible es casi exclusivamente de naturaleza institucional: ayudas, legislación, etcétera; $c$ ) entre la población, la información sobre la realidad del sector es reducida y se asocia, casi exclusivamente, a una imagen negativa de pobreza, incultura y subdesarrollo.

Después de este análisis tomaron varias decisiones: $a$ ) ampliar el espectro de población alcanzado por el periódico del sector y aumentar la regularidad de su aparición; $b$ ) crear un recurso informativo específico para el sector en línea, que proporcione información general sobre su realidad (cultura, vida de cada día, información actualizada); c) definir una estrategia de valorización para el sector, basada en el desarrollo del fenómeno de la información, que contribuya a mejorar el nivel informativo de la población en general; $d$ ) crear recursos informativos en formatos y contextos diversos.

Scire. $14: 1$ (en.-jun. 2008) 75-87. ISSN 1135-3716. 
Para conseguirlo se crearon o mejoraron diversos recursos informativos: el periódico Voz dos Marítimos, el portal del sector pesquero de las Azores (www.pescas.net) y el CD-ROM Iniciativas empresariales en las industrias pesqueras y el mar, entre otros. A partir de esta experiencia concluimos que, para desarrollar eficientemente el sistema de información externo de este tipo de proyectos, es esencial la puesta en marcha de uno de los recursos siguientes (o todos): un periódico sectorial, integrado en publicaciones de alcance más amplio, con una distribución que permite llegar a todas las localidades azoreanas; un portal sectorial (www.pescas.net) que utilizan preferentemente los profesionales conectados con el sector y también la población en general, con una media de 150000 visitas mensuales; una base de datos temática para el sector en cuestión, disponible en formato CD-ROM y en línea (www.pescas.net/iep), utilizada como herramienta formativa para la aproximación a las tecnologías, pero también para ampliar la percepción de la realidad y las perspectivas sociales y económicas del sector.

Finalmente, es esencial trabajar todas estas herramientas en una forma integrada, de tal manera que el contenido de un(os) recurso(s) pueda revalorizar siempre el contenido del otro(s).

\section{Perspectivas}

La familia de productos Estrategias y recursos de información para la gerencia de proyectos compartidos y sectoriales fue resultado de una experiencia práctica muy rica, siempre desafiante: la de articular información, gestión de la información y tecnología en un proyecto europeo cuyo principal objetivo se centraba en el desarrollo de un sector socialmente desfavorecido y empobrecido y con muchas prioridades de intervención. A primera vista, la gestión de la información parece algo alejada de las necesidades fundamentales de dignidad, pero hay que tener en cuenta algunas cuestiones que se exponen a continuación.

En primer lugar, la información es lo que nos conecta como seres humanos, nos permite conocernos, compartir.

Hoy, con Internet, además de la "percepción universal" de la biblioteca infinita de Borges, existe como nunca la posibilidad concreta de un aumento exponencial de la comunicación entre los pueblos (y no solamente entre los gobiernos de los pueblos).

La información no es, no puede ser, para una minoría que continúe perpetuando unos conceptos elitistas de información y de conocimiento que hasta hoy han venido determinando lo que es el saber/información "más" o "menos" importante; se trata de una cuestión de todas las personas y de todas las comunidades.

Poseer información sobre cómo pescar tiene tanto valor intrínseco como tenerla, por ejemplo, para la construcción de una base de datos. 
Las tecnologías de la información están asociadas a la comprensión de un lenguaje que, cada vez más, es como leer y escribir.

Las potencialidades de la información y de la gestión de la información para descubrir, valorar y compartir experiencias sobre y entre las poblaciones son muy grandes.

Los partenariados y redes que conectan amplios espacios sociales y geográficos constituyen entornos de desarrollo social muy interesantes y cada vez más comunes. La información y la gestión de la información son factores críticos para la existencia de redes efectivas y para su transformación en verdaderas comunidades de prácticas.

Según lo expuesto, una vez discutido todo esto en el partenariado, trabajamos durante dos años sobre la información y la gestión de la información, de forma que no solamente fuesen útiles para los objetivos del proyecto, sino que también los potenciasen. Tuvimos también la oportunidad de compartir y discutir las experiencias y los resultados en diversos locales y con muchas personas de las comunidades de las Azores, en el espacio europeo e incluso en la Comisión Europea, que los ha propuesto como ejemplo de buenas prácticas. El interés de los resultados y la curiosidad por comprenderlos y sistematizarlos con el apoyo de una investigación de tipo teórico-práctico han originado un proyecto de investigación y doctorado en el que estoy trabajando en la actualidad y donde espero poder profundizar en las sensaciones percibidas en la práctica y extraer resultados capaces de mejorar y agilizar la información para el trabajo en red, lo que considero, cada vez más, como una de las nuevas y más fuertes potencialidades del entorno social contemporáneo.

\section{Referencias}

Abadal Falgueras, Ernest (2004). Gestión de proyectos en información y documentación. Gijón: Trea.

Borko, Harold (1968). Information Science, what is it? // American Documentation. 19:1 (1968) 3-5.

Castells, Manuel (1996). The information age: economy, society and culture, vol. 1: The rise of the network society. Oxford: Blackwells, 1996. Trad. esp.: Castells, Manuel (19971998). La era de la información: economía sociedad y cultura, vol. 1: La sociedad red. Madrid: Alianza, 1997-1998.

Choo, Chun Wei (2003). Gestão de informação para a organização inteligente. A arte de explorar o meio ambiente. Lisboa: Caminho, 2003.

Delgado López-Cózar, Emilio (2002). La investigación en biblioteconomía y documentación. Gijón: Trea.

García Jiménez, Antonio (2002). Organización y gestión del conocimiento en la comunicación. Gijón: Trea.

Ribeiro, Fernanda; Silva, Armando Malheiro de (2004). A avaliação da informação: uma operação metodológica. // Páginas A\&B: Arquivos e Bibliotecas. 14 (2004) 7-37.

Scire. $14: 1$ (en.-jun. 2008) 75-87. ISSN 1135-3716. 
Silva, Armando Malheiro da, et álii (1998). Arquívistica: Teoria e prática de uma ciência da informação, vol. 1. Oporto: Afrontamento ("Biblioteca das Ciências do Homem. Plural”, 4), 1998.

\section{Apéndice: principales pantallas del sistema}

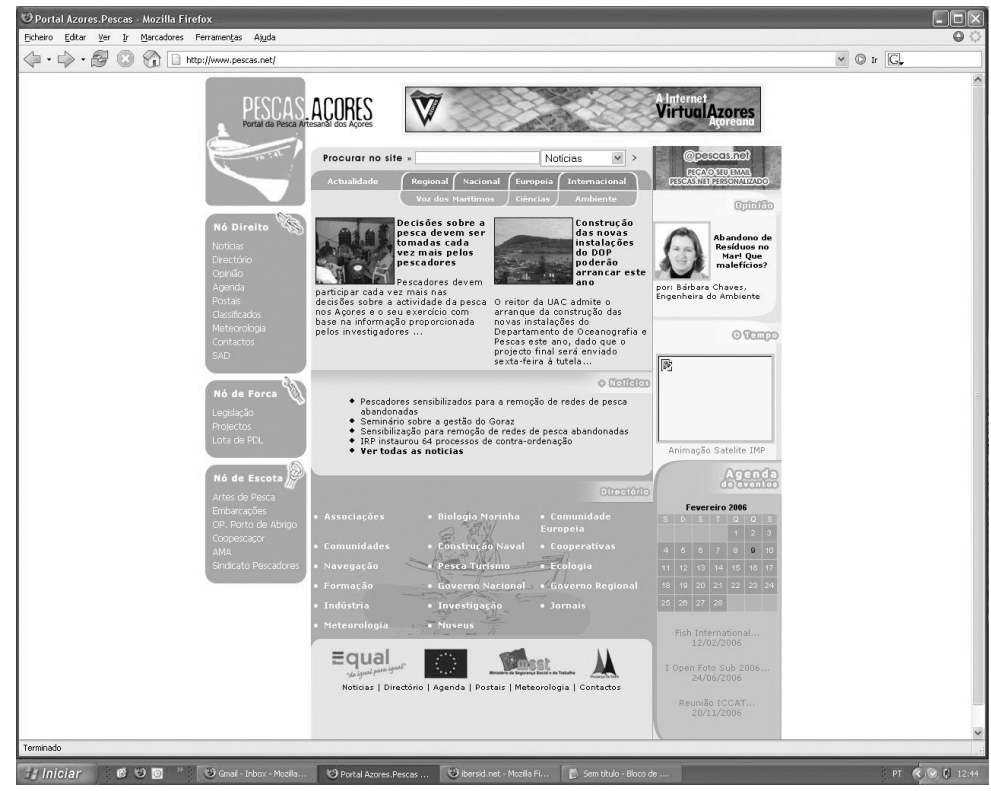

Figura 1. 


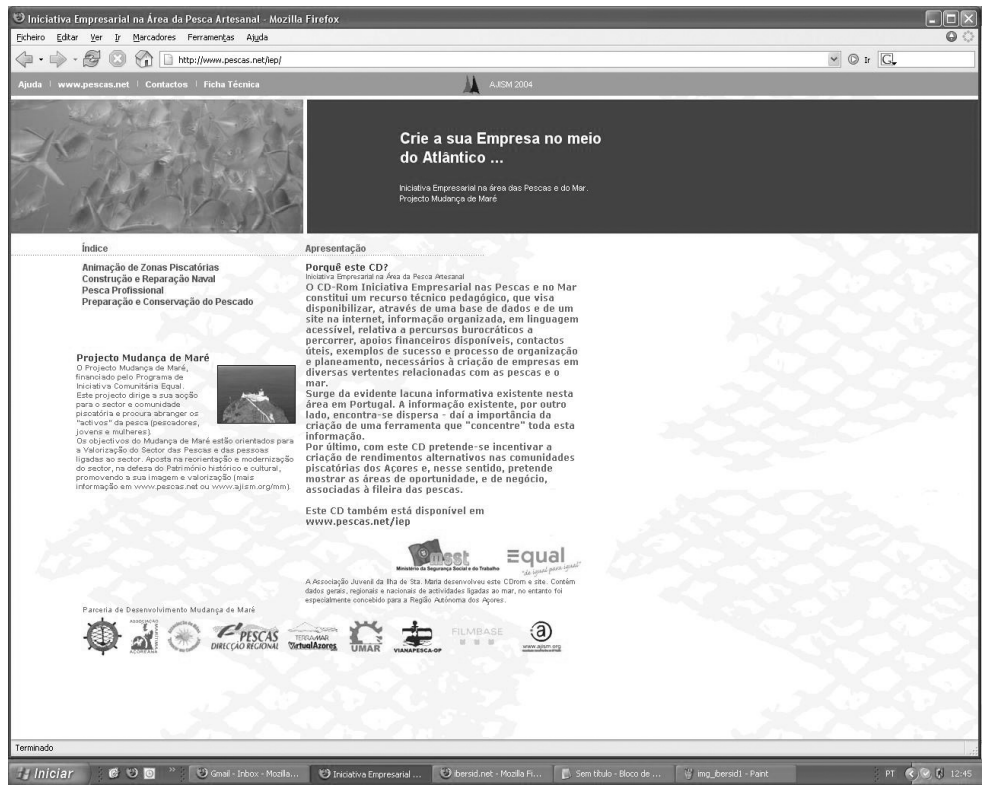

Figura 2.

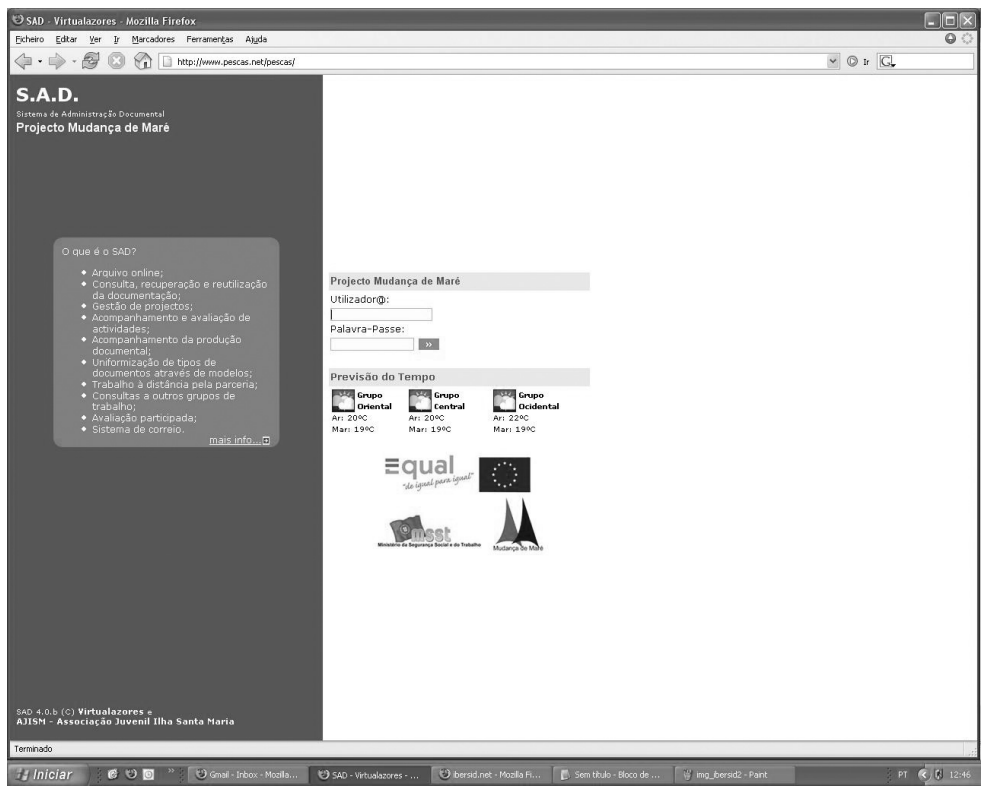

Figura 3.

Scire. $14: 1$ (en.-jun. 2008) 75-87. ISSN 1135-3716. 


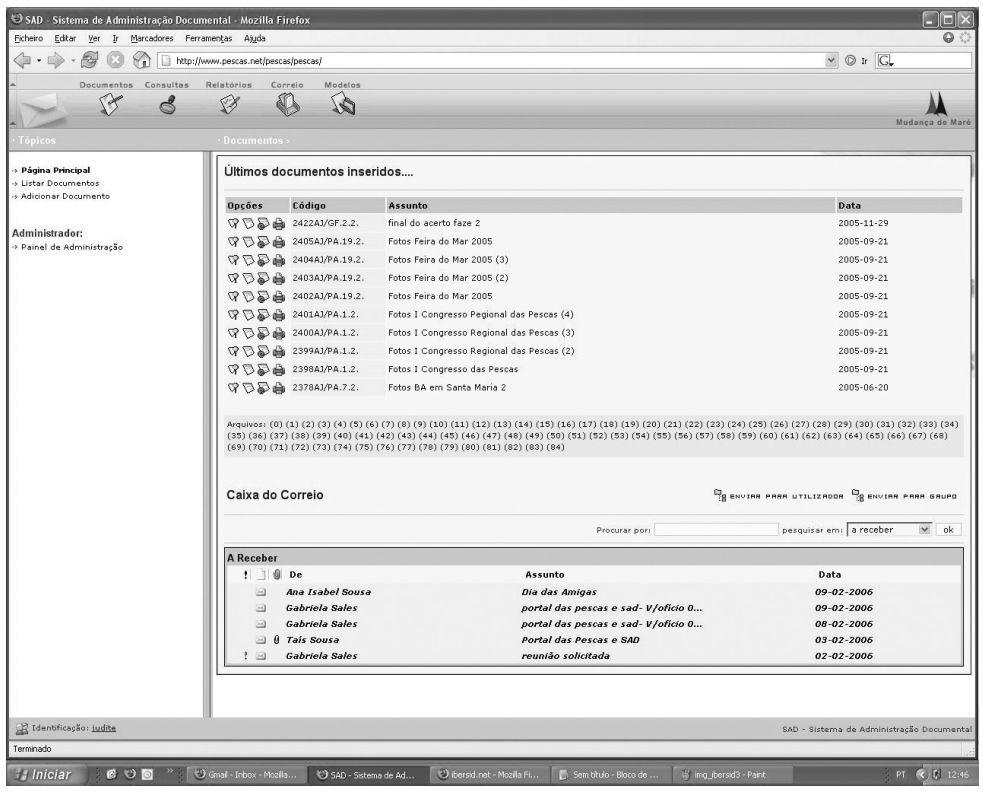

Figura 4. 